\title{
Integrated Genomic Analysis Highlights the Impaired PI3K-Akt Signaling Pathway in Alzheimer's Disease
}

\author{
Mozhgan Jirehnezhadyan ${ }^{1}$, Reza Fahimi ${ }^{2}$, Yaser Asefi ${ }^{3, *}$ \\ 1 Department of animal biology, Arvandan Non-Profit Higher Education Institute, Khorramshahr, Iran; \\ mozhgan201469@gmail.com (M.J.); \\ 2 Medical Research Center, Kateb University, Kabul, Afghanistan; fahimi90@gmail.com (R.F.); \\ 3 Department of Genetics, Ahar Branch, Islamic Azad University, Ahar, Iran; asefiyaser65@gmail.com (Y.A.); \\ * Correspondence: asefiyaser65@gmail.com (Y.A.);
}

Scopus Author ID 57216565685

Received: 13.05.2021; Revised: 10.06.2021; Accepted: 12.06.2021; Published: 18.06.2021

\begin{abstract}
Alzheimer's disease (AD), a leading cause of dementia, remained incurable, despite many advances in our knowledge about AD pathogenesis, underlying mechanisms are poorly understood. Transcriptome analysis showed efficiency in exploring these mechanisms; however, data are generated at a higher pace than interpreted and are almost inconsistent. Therefore we performed this meta-analysis to extract new knowledge from existing data and find the mechanisms involved in AD. Five temporal cortex transcriptomics datasets from 187 AD patients and 167 healthy controls were analyzed. Our analysis showed that the PI3K-Akt signaling pathway is significantly impaired in AD brains and was common among all datasets. Moreover, miRs targeting genes involved in the PI3K-Akt signaling pathway were identified. In conclusion, our results highlight the impaired PI3K-Akt signaling pathway in $\mathrm{AD}$ and suggested related miRs as the potential targets for early treatment and diagnosis of AD.
\end{abstract}

Keywords: Alzheimer's disease; meta-analysis; transcriptome; PI3K/Akt signaling pathway

(C) 2021 by the authors. This article is an open-access article distributed under the terms and conditions of the Creative Commons Attribution (CC BY) license (https://creativecommons.org/licenses/by/4.0/).

\section{Introduction}

Alzheimer's disease (AD) is a progressive and irreversible neurodegenerative disease that is characterized by the presence of senile plaques (SPs) and neurofibrillary tangles (NFTs) $[1,2]$. AD is the leading cause of dementia, accounting for about $60-70 \%$ of dementia cases, currently, around 50 million people are living with dementia globally, which is estimated to reach 82 million in 2030 and 152 in 2050 unless preventive strategies are found [3,4]. Two well-established risk factors for developing AD are Apolipoprotein E (ApoE) polymorphism and aging [5]. Despite many advances in our knowledge about AD pathogenesis, the underlying mechanisms are poorly understood and recent disappointing clinical outcomes of targeting two well-established AD pathological hallmarks, including NFTs and SPs have challenged our narrow understanding of AD pathogenesis [6,7].

The development of high-throughput technologies enabled us to investigate the changes at molecular levels corresponding to disease development and progression, however, huge amount of data are generated through these technologies at a higher pace than they are interpreted [8,9]. Microarray analysis as one of these high-throughput technologies has gained a great deal of attention among researchers from different fields, including AD. A growing number of research studies have been performed to elucidate the gene expression alteration in AD brains $[10,11]$. 
While microarray analysis proved to be an efficient tool to explore the AD-related changes at the gene expression level, however reproducibility of microarray analyses has always been questionable. Inconsistent results are commonly observed across different studies, furthermore considering the multifactorial nature of $\mathrm{AD}$, different genetic backgrounds and lifestyles could affect these molecular changes across AD patients [12-14]. In this regard, a meta-analysis that combines the outcomes of several studies appeared as powerful tools to reduce the heterogeneity among published results and define the most reliable changes [15-19]. Currently, there are several meta-analyses on gene expression data from different brain regions of AD patients; including $\mathrm{Li}$ and colleagues meta-analysis in 2015 on six studies from the frontal cortex of AD patients [16], Moradifard and colleagues meta-analysis in 2018, which included data from six individual studies and further a sub-meta-analysis on the hippocampus and entorhinal cortex [20], and recently a meta-analysis on CA1 of the hippocampus DEGs by Hosseinian and colleagues in 2020 [12]. However, meta-analyses on other brain regions are not available. Therefore we herein performed a comprehensive meta-analysis on the temporal cortex microarray datasets from AD patients.

\section{Materials and Methods}

\subsection{Search strategy and data collection.}

A comprehensive search through Gene Expression Omnibus (GEO) was performed to find all eligible datasets from inception up to March 2021. "Alzheimer" and "temporal" were used as keywords and three filters including Homo sapiens, Series, and Expression profiling by array were employed. Differentially expressed genes (DEGs) between AD patients and healthy controls were obtained using the GEO2R tool and DEGs with adjusted $\mathrm{P}$ value $<0.05$ were considered significant.

\subsection{Integrated genomic analyses.}

Herein for our meta-analysis, we used R package RobustRankAggreg. Unlike Rank Aggregation (RA) that detect the closest list to the input lists, RRA generates a relevant list of even irrelevant and incomplete input lists [21]. Robust DEGs for each brain region were considered significant if the Bonferroni-corrected $\mathrm{p}$-value was $<0.05$. Moreover, common genes between at least two datasets (common DEGs) were identified.

\subsection{Enriched pathways.}

For pathway analysis, we submitted Robust DEGs list in The Database for Annotation, Visualisation, and Integrated Discovery (DAVID) and selected KEGG-pathways with Benjamini-corrected p-value less than 0.05, furthermore, pathways for common DEGs were also identified [22-24]. Finally, we found the pathways that were significant in both robust DEGs and common DEGs.

\subsection{Targeting miRs.}

To find miRs targeting genes involved in PI3K-Akt signaling pathway, GeneSet2miRNA (GS2M) was used in www.bioprofiling.de, which employed 11 prediction programs. ENTREZ GENE IDs for both Robust and common genes were submitted in GS2M 
and miRs with Mont Carlo-corrected p-value less than 0.05 were considered statistically significant [25-27].

\section{Results and Discussion}

\subsection{Search results and differentially expressed genes.}

Our search through GEO yielded 24 datasets, of those 5 datasets including GSE118553, GSE5281, GSE36980, GSE122063, GSE132903 containing 187 AD patients and 167 healthy controls were included in our study. The basic characteristics of included studies are summarized in Table 1. DEGs between the control and AD group were identified using the GEO2R tool, where P-values less than 0.05 were considered significant.

Table 1. Basic characteristics of included studies.

\begin{tabular}{c|c|c|c|c|c} 
Datasets & \multirow{2}{*}{ Country } & $\begin{array}{c}\text { Number of } \\
\text { AD/CTR }\end{array}$ & $\begin{array}{c}\text { Age (yrs.) } \\
\text { AD/CTR }\end{array}$ & $\begin{array}{c}\text { Postmortem interval (hours) } \\
\text { AD/CTR }\end{array}$ & Reference \\
\hline GSE118553 & UK & $52 / 27$ & $82.9 \pm 8.7 / 70.6 \pm 15.9$ & $39.9 \pm 21.3 / 37.1 \pm 20.7$ & {$[28]$} \\
\hline GSE5281 & USA & $33 / 14$ & $79.9 \pm 6.9 / 79.8 \pm 9.1$ & $2.5 / 2.5$ & {$[29,30]$} \\
\hline GSE36980 & Japan & $26 / 62$ & $83.0 \pm 5.7 / 83.0 \pm 5.7$ & - & {$[31,32]$} \\
\hline GSE122063 & USA & $12 / 11$ & $80.9 \pm 7.4 / 78.6 \pm 8.5$ & $8.0 \pm 4.0 / 9.0 \pm 3.0$ & {$[33]$} \\
\hline GSE132903 & USA & $97 / 98$ & $85.02 \pm 6.75 / 84.98 \pm 6.90$ & - & {$[10]$}
\end{tabular}

3.2. Results of integrated genomic analyses.

RRA analyses for identification of robust DEGs between datasets yielded a set of up and down-regulated genes that top five up and down-regulated robust genes are given in Table 2, furthermore, common genes between included studies were obtained using Venn diagram (Figure 1).
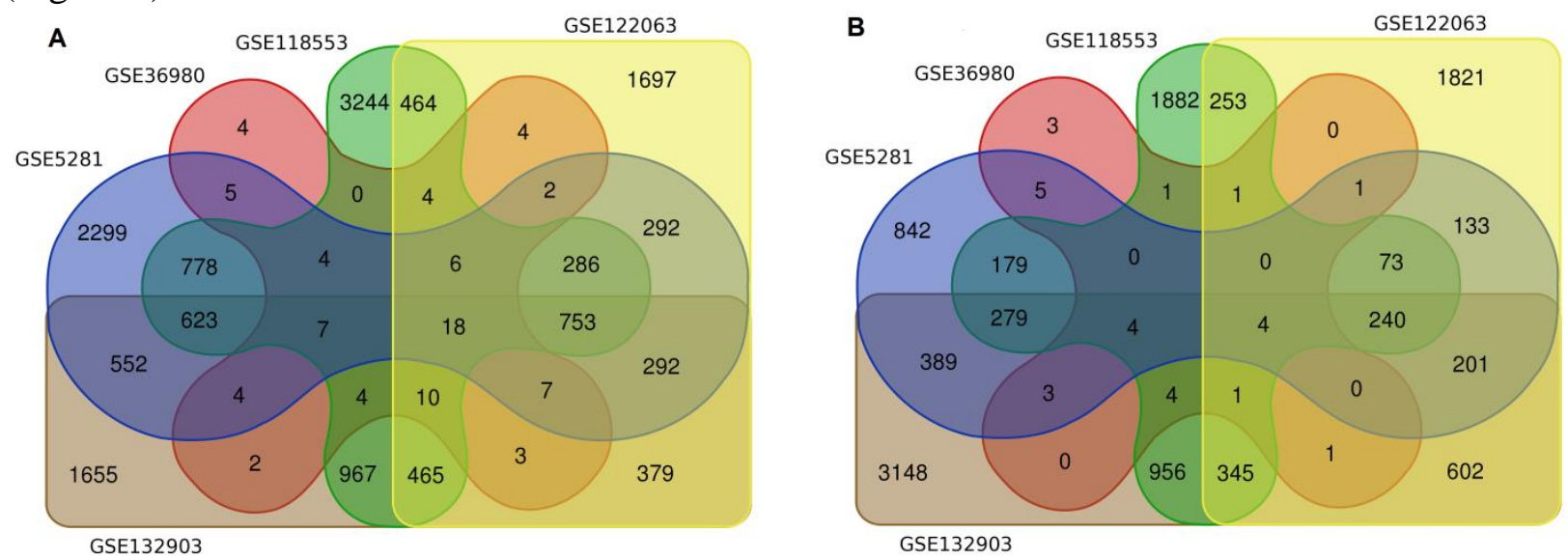

Figure 1. Common A) down-regulated and B) up-regulated genes between included studies, developed by Van der Peer Lab [34].

Table 2. Top five up and down-regulated robust genes.

\begin{tabular}{|c|c|c|}
\hline Gene Symbol & Full name & Score \\
\hline \multicolumn{3}{|c|}{ Robust up-regulated genes } \\
\hline APLNR & Apelin Receptor & $4.251491 \mathrm{e}-09$ \\
\hline ITPKB & Inositol-Trisphosphate 3-Kinase $\mathrm{B}$ & $3.801006 \mathrm{e}-06$ \\
\hline AEBP1 & AE Binding Protein 1 & $1.391380 \mathrm{e}-05$ \\
\hline SERPINA3 & Serpin Family A Member 3 & $4.576840 \mathrm{e}-05$ \\
\hline ANLN & Anillin Actin Binding Protein & $1.279258 \mathrm{e}-04$ \\
\hline \multicolumn{3}{|c|}{ Robust down-regulated genes } \\
\hline VSNL1 & Visinin Like 1 & $4.370754 \mathrm{e}-08$ \\
\hline
\end{tabular}




\begin{tabular}{l|l|l}
\hline Gene Symbol & Full name & Score \\
\hline CARTPT & CART Prepropeptide & $2.274075 \mathrm{e}-07$ \\
\hline NPTX2 & Neuronal Pentraxin 2 & $2.885267 \mathrm{e}-07$ \\
\hline VGF & VGF Nerve Growth Factor Inducible & $6.008304 \mathrm{e}-07$ \\
\hline SYT13 & Synaptotagmin 13 & $7.091239 \mathrm{e}-7$
\end{tabular}

3.3. Pathways enriched by differentially expressed genes.

DAVID was used to find pathways enriched by robust and common DEGs, the top three pathways based on a number of genes and P-Values are given in Table 3, interestingly the PI3K-Akt signaling pathway was a top pathway enriched by both robust genes and common DEGs. Both phosphoinositide 3-kinase (PI3K) and protein kinase B (PKB or Akt) are kinases.

Table 3. Three top pathways enriched by up and down-regulated robust and common genes.

\begin{tabular}{|c|c|c|}
\hline Pathway & Number of genes & P-Value \\
\hline \multicolumn{3}{|l|}{ Robust up-regulated genes } \\
\hline PI3K-Akt signaling pathway & 10 & $1.70 \mathrm{e}-2$ \\
\hline HTLV-I infection & 8 & $2.60 \mathrm{e}-2$ \\
\hline Rap1 signaling pathway & 7 & $3.30 \mathrm{e}-2$ \\
\hline \multicolumn{3}{|l|}{ Robust down-regulated genes } \\
\hline Neuroactive ligand-receptor interaction & 13 & $4.80 \mathrm{e}-4$ \\
\hline GABAergic synapse & 12 & $2.20 \mathrm{e}-8$ \\
\hline Retrograde endocannabinoid signaling & 12 & $1.40 \mathrm{e}-7$ \\
\hline \multicolumn{3}{|l|}{ Common up-regulated genes } \\
\hline Pathways in cancer & 140 & $8.80 \mathrm{e}-14$ \\
\hline PI3K-Akt signaling pathway & 114 & $4.40 \mathrm{e}-9$ \\
\hline Focal adhesion & 80 & $3.50 \mathrm{e}-10$ \\
\hline \multicolumn{3}{|l|}{ Common down-regulated genes } \\
\hline Metabolic pathways & 444 & $4.60 \mathrm{e}-7$ \\
\hline Huntington's disease & 108 & $1.20 \mathrm{e}-13$ \\
\hline Alzheimer's disease & 102 & $7.00 \mathrm{e}-16$ \\
\hline
\end{tabular}

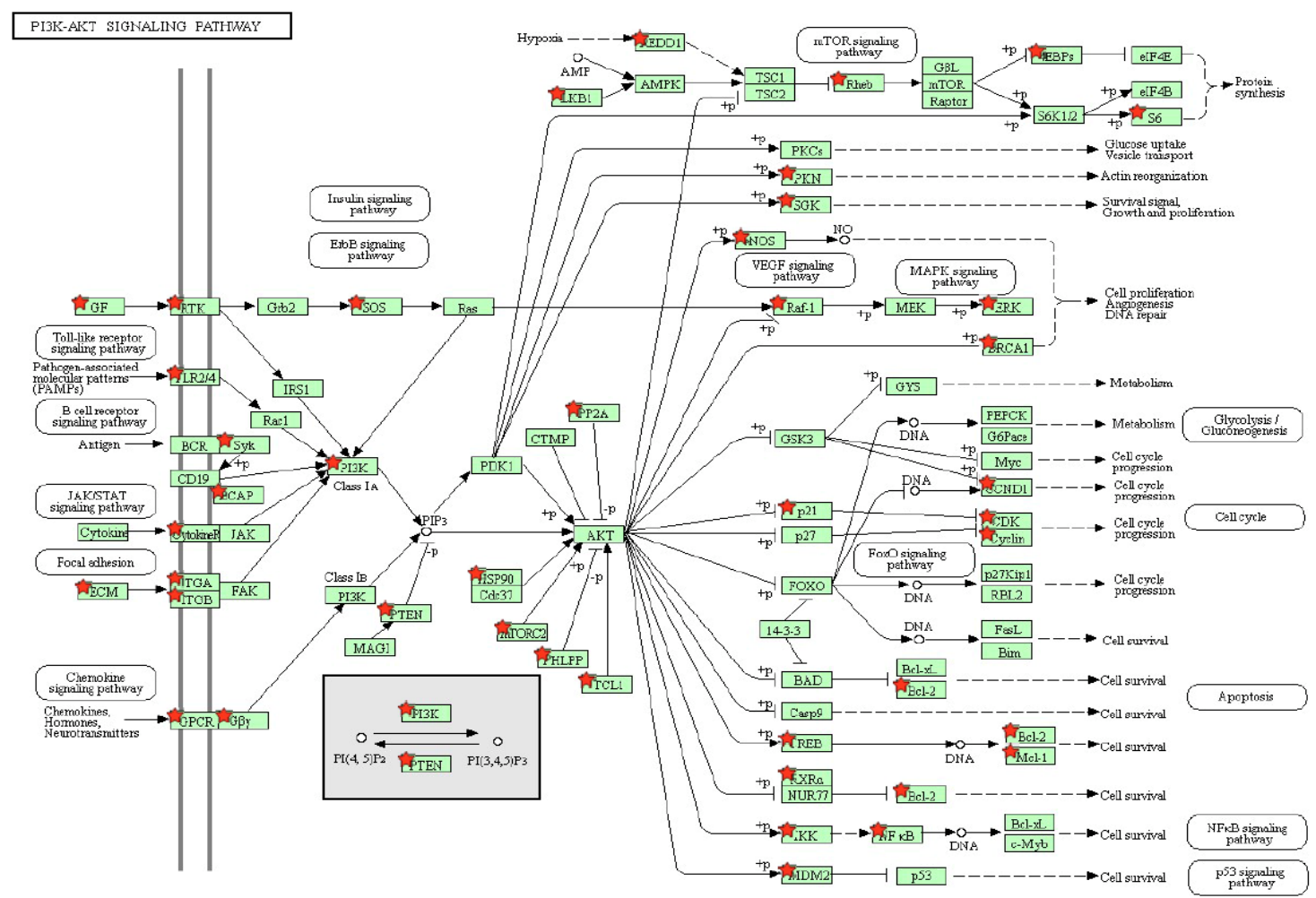

Figure 2. PI3K-Akt signaling pathway enriched by both robust and common genes that are marked by red stars reproduced with copyright permission from KEGG [24,35]. 
3.4. miRs targeting genes involved in the PI3K-Akt signaling pathway.

Results of miRs analyses for genes involved in PI3K-Akt signaling pathway using GS2M yielded $38 \mathrm{miRs}$, of those $7 \mathrm{miRs}$ had the P-value less than 0.05 and showed to be statistically significant. The top three miRs based on a number of target genes and P-values are given in table 4; Including HSA-MIR-29B.4, HSA-MIR-29B.5 and HSA-MIR-29C.5 that target 21,17 and 15 genes, respectively.

Table 4. Top three predicted miRs targeting genes involved in the PI3K-Akt signaling pathway

\begin{tabular}{|c|c|c|}
\hline $\operatorname{miR}$ & P-value & Number of targeting genes \\
\hline HSA-MIR-29B.4 & $7.57229967044858 \mathrm{e}-05$ & $\begin{array}{l}\text { (21): CDK6, CDK2, COL1A2, COL4A1, COL4A4, } \\
\text { COL4A5, COL6A3, COL11A1, ITGA6, ITGB1, LAMC1, } \\
\text { LAMA2, MCL1, PDGFRB, PTEN, SGK1, COL5A3, } \\
\text { GNG12, PDFGC, THBS2, ITGA11. }\end{array}$ \\
\hline HSA-MIR-29B.5 & $1.28970003513928 \mathrm{e}-06$ & $\begin{array}{l}\text { (17): CDK6, COL1A2, COL4A1, COL4A4, COL4A5, } \\
\text { COL6A3, COL11A1, ITGA6, ITGB1, LAMC1, MCL1, } \\
\text { PDGFRB, PTEN, SGK1, COL5A3, GNG12, PDFGC. }\end{array}$ \\
\hline HSA-MIR-29C.5 & 0.000126067421820976 & $\begin{array}{l}\text { (15): CDK6, COL1A2, COL4A1, COL4A4, COL4A5, } \\
\text { COL6A3, COL11A1, ITGB1, LAMC1, MCL1, PDGFRB, } \\
\text { PTEN, SGK1, COL5A3, GNG12. }\end{array}$ \\
\hline
\end{tabular}

Herein, we have performed an integrated genomic analysis on microarray datasets from 187 AD patients and 167 healthy controls, our results showed significant up-regulation of genes involved in the PI3K-Akt signaling pathway in AD brains compared to healthy controls.

A PI3K-Akt signaling pathway is an essential modulator of insulin effects and its impairment is a key pathological event in type 2 diabetes (T2D), recently impaired PI3K-Akt signaling pathway has been reported in AD patients and has been proposed as a mechanism linking $\mathrm{AD}$ and T2D; however results are almost inconsistent, while most of the reports reported down-regulation of this pathway in $\mathrm{AD}$ patients [36,37], a few numbers of evidence indicated up-regulation of this pathway in AD and causing cognitive impairment $[38,39]$, moreover, there is also some evidence indicating up-regulation of this pathway in an early stage of $\mathrm{AD}$, possibly as a compensatory response which is then down-regulated upon $\mathrm{AD}$ progression [37,39,40]. PI3K and Akt are two kinases that are involved in different signaling pathways, including glycogen synthase kinase 3 beta (GSK-3 $\beta$ ) inactivation; GSK-3 $\beta$ is responsible for tau protein phosphorylation and played a key role in AD pathogenesis, GSK$3 \beta$ is usually inactivated through phosphorylation by Akt, which its activation is modulated by PI3K. Therefore, the mechanism for the role of the PI3K-Akt signaling pathway in AD pathogenesis is based on its deregulation leading to GSK-3 $\beta$ activation, which in turn increased tau phosphorylation [36]. However, the results of this study showed the up-regulation of the PI3K-Akt signaling pathway, which is in contrast to this mechanism, which is possibly a part of the compensatory response in an early stage of AD. Moreover, herein we have identified miRs targeting genes involved in the PI3K-Akt signaling pathway, which may be downregulated in AD patients, specifically in early stages, although experimental studies are needed to confirm our results, these miRs may be potential therapeutic targets and biomarkers for AD treatment or early diagnosis.

\section{Conclusions}

In conclusion, in this study, we reached from thousands of differentially expressed genes in $\mathrm{AD}$ brain to tens of robust genes and tens of common genes between at least two 
studies and showed the impaired PI3K-Akt signaling pathway in AD, subsequently, the miRs analysis showed the potential miRs targeting genes involved in PI3K-Akt signaling pathway with potential application as therapeutic and diagnostic biomarkers in AD.

\section{Funding}

This research received no external funding.

\section{Acknowledgments}

We want to express our sincere gratitude to the anonymous reviewers for their valuable comments and suggestions to improve the paper's quality.

\section{Conflicts of Interest}

\section{The authors declare no conflict of interest}

\section{References}

1. Saez-Atienzar, S.; Masliah, E. Cellular senescence and Alzheimer disease: the egg and the chicken scenario. Nature Reviews Neuroscience 2020, 21, 433-444, https://doi.org/10.1038/s41583-020-0325-Z.

2. Janelidze, S.; Berron, D.; Smith, R.; Strandberg, O.; Proctor, N.K.; Dage, J.L.; Stomrud, E.; Palmqvist, S.; Mattsson-Carlgren, N.; Hansson, O. Associations of plasma phospho-Tau217 levels with tau positron emission tomography in early Alzheimer disease. JAMA neurology 2021, 78, 149-156, https://doi.org/10.1001/jamaneurol.2020.4201.

3. WHO. Dementia fact sheet December 2017;. World Health Organisation 2020, https://www.who.int/newsroom/fact-sheets/detail/dementia

4. Abyadeh, M.; Djafarian, K.; Heydarinejad, F.; Alizadeh, S.; Shab-Bidar, S. Association between Apolipoprotein E Gene Polymorphism and Alzheimer's Disease in an Iranian Population: A Meta-Analysis. Journal of Molecular Neuroscience 2019, 69, 557-562, https://doi.org/10.1007/s12031-019-01381-1.

5. Fyfe, I. Epigenetics links ageing with Alzheimer disease. Nature Reviews Neurology 2018, 14, 254-254, https://doi.org/10.1038/nrneurol.2018.36.

6. Selkoe, D.J. Alzheimer disease and aducanumab: adjusting our approach. Nature Reviews Neurology 2019, 15, 365-366, https://doi.org/10.1038/s41582-019-0205-1.

7. Cummings, J.; Feldman, H.H.; Scheltens, P. The "rights" of precision drug development for Alzheimer's disease. Alzheimer's research \& therapy 2019, 11, 1-14, https://doi.org/10.1186/s13195-019-0529-5.

8. Abyadeh, M.; Meyfour, A.; Gupta, V.; Zabet Moghaddam, M.; Fitzhenry, M.J.; Shahbazian, S.; Hosseini Salekdeh, G.; Mirzaei, M. Recent Advances of Functional Proteomics in Gastrointestinal Cancers-a Path towards the Identification of Candidate Diagnostic, Prognostic, and Therapeutic Molecular Biomarkers. International Journal of Molecular Sciences 2020, 21, 8532, https://doi.org/10.3390/ijms21228532.

9. Wingo, A.P.; Liu, Y.; Gerasimov, E.S.; Gockley, J.; Logsdon, B.A.; Duong, D.M.; Dammer, E.B.; Robins, C.; Beach, T.G.; Reiman, E.M. Integrating human brain proteomes with genome-wide association data implicates new proteins in Alzheimer's disease pathogenesis. Nature Genetics 2021, 53, 143-146, https://doi.org/10.1038/s41588-020-00773-z.

10. Piras, I.S.; Krate, J.; Delvaux, E.; Nolz, J.; Mastroeni, D.F.; Persico, A.M.; Jepsen, W.M.; Beach, T.G.; Huentelman, M.J.; Coleman, P.D. Transcriptome changes in the Alzheimer's disease middle temporal gyrus: importance of RNA metabolism and mitochondria-associated membrane genes. Journal of Alzheimer's Disease 2019, 70, 691-713, https://doi.org/10.3233/JAD-181113.

11. Belonwu, S.; Li, Y.; Bunis, D.; Rao, A.A.; Solsberg, C.W.; Oskotsky, T.; Taubes, A.; Grone, B.; Zalocusky, K.; Fragiadakis, G. Single-cell transcriptomic analysis elucidates APOE genotype specific changes across cell types in two brain regions in Alzheimer's disease. 2021, https://doi.org/10.21203/rs.3.rs-291648/v1.

12. Hosseinian, S.; Arefian, E.; Rakhsh-Khorshid, H.; Eivani, M.; Rezayof, A.; Pezeshk, H.; Marashi, S.-A. A meta-analysis of gene expression data highlights synaptic dysfunction in the hippocampus of brains with Alzheimer's disease. Scientific reports 2020, 10, 1-9, https://doi.org/10.1038/s41598-020-64452-z.

13. Noori, A.; Mezlini, A.M.; Hyman, B.T.; Serrano-Pozo, A.; Das, S. Systematic review and meta-analysis of human Transcriptomics reveals Neuroinflammation, deficient energy metabolism, and Proteostasis failure across Neurodegeneration. Neurobiology of Disease 2020, 105225, https://doi.org/10.1016/j.nbd.2020.105225.

14. Schwartzentruber, J.; Cooper, S.; Liu, J.Z.; Barrio-Hernandez, I.; Bello, E.; Kumasaka, N.; Young, A.M.; Franklin, R.J.; Johnson, T.; Estrada, K. Genome-wide meta-analysis, fine-mapping and integrative 
prioritization implicate new Alzheimer's disease risk genes. Nature Genetics 2021, 53, 392-402, https://doi.org/10.1038/s41588-020-00776-w.

15. Asefi, Y.; Gohari Mahmoudabad, A.; Habibian Sezavar, A.; Mirshahvaladi, S.; Abyadeh, M.; Abyareh, M. Association between maternal cadmium exposure and preterm birth: a meta-analysis. International Journal of Environmental Health Research 2020, 1-10, https://doi.org/10.1080/09603123.2020.1789947.

16. Li, X.; Long, J.; He, T.; Belshaw, R.; Scott, J. Integrated genomic approaches identify major pathways and upstream regulators in late onset Alzheimer's disease. Scientific reports 2015, 5, 1-12, https://doi.org/10.1038/srep12393.

17. Abyadeh, M.; Heydarinejad, F.; Khakpash, M.; Asefi, Y.; Shab-Bidar, S. Association of Apolipoprotein E gene polymorphism with Preeclampsia: a meta-analysis. Hypertension in pregnancy 2020, 39, 196-202, https://doi.org/10.1080/10641955.2020.1753068.

18. Sun, Z.; Tan, J.; Zhao, M.; Peng, Q.; Zhou, M.; Zuo, S.; Wu, F.; Li, X.; Dong, Y.; Xie, M. Integrated genomic analysis reveals regulatory pathways and dynamic landscapes of the tRNA transcriptome. Scientific reports 2021, 11, 1-16, https://doi.org/10.1038/s41598-021-83469-6.

19. García-Gómez, B.E.; Salazar, J.A.; Nicolás-Almansa, M.; Razi, M.; Rubio, M.; Ruiz, D.; Martínez-Gómez, P. Molecular Bases of Fruit Quality in Prunus Species: An Integrated Genomic, Transcriptomic, and Metabolic Review with a Breeding Perspective. International Journal of Molecular Sciences 2021, 22, 333, https://doi.org/10.3390/ijms22010333.

20. Moradifard, S.; Hoseinbeyki, M.; Ganji, S.M.; Minuchehr, Z. Analysis of microRNA and gene expression profiles in Alzheimer's disease: a meta-analysis approach. Scientific reports 2018, 8, 1-17, https://doi.org/10.1038/s41598-018-20959-0.

21. Kolde, R.; Laur, S.; Adler, P.; Vilo, J. Robust rank aggregation for gene list integration and meta-analysis. Bioinformatics 2012, 28, 573-580, https://doi.org/10.1093/bioinformatics/btr709.

22. Sherman, B.T.; Lempicki, R.A. Systematic and integrative analysis of large gene lists using DAVID bioinformatics resources. Nature protocols 2009, 4, 44, https://doi.org/10.1038/nprot.2008.211.

23. Huang, D.W.; Sherman, B.T.; Lempicki, R.A. Bioinformatics enrichment tools: paths toward the comprehensive functional analysis of large gene lists. Nucleic acids research 2009, 37, 1-13, https://doi.org/10.1093/nar/gkn923.

24. Kanehisa, M.; Goto, S. KEGG: kyoto encyclopedia of genes and genomes. Nucleic acids research 2000, 28 , 27-30, https://doi.org/10.1093/nar/28.1.27.

25. Antonov, A.V.; Dietmann, S.; Wong, P.; Lutter, D.; Mewes, H.W. GeneSet2miRNA: finding the signature of cooperative miRNA activities in the gene lists. Nucleic acids research 2009, 37, W323-W328, https://doi.org/10.1093/nar/gkp313.

26. Xiao, F.; Zuo, Z.; Cai, G.; Kang, S.; Gao, X.; Li, T. miRecords: an integrated resource for microRNA-target interactions. Nucleic acids research 2009, 37, D105-D110, https://doi.org/10.1093/nar/gkn851.

27. Aghaee-Bakhtiari, S.H.; Arefian, E.; Lau, P. miRandb: a resource of online services for miRNA research. Briefings in bioinformatics 2018, 19, 254-262, https://doi.org/10.1093/bib/bbw109.

28. Patel, H.; Hodges, A.K.; Curtis, C.; Lee, S.H.; Troakes, C.; Dobson, R.J.; Newhouse, S.J. Transcriptomic analysis of probable asymptomatic and symptomatic alzheimer brains. Brain, behavior, and immunity 2019, 80, 644-656, https://doi.org/10.1016/j.bbi.2019.05.009.

29. Liang, W.S.; Dunckley, T.; Beach, T.G.; Grover, A.; Mastroeni, D.; Ramsey, K.; Caselli, R.J.; Kukull, W.A.; McKeel, D.; Morris, J.C. Altered neuronal gene expression in brain regions differentially affected by Alzheimer's disease: a reference data set. Physiological genomics 2008, 33, 240-256, https://doi.org/10.1152/physiolgenomics.00242.2007.

30. Liang, W.S.; Dunckley, T.; Beach, T.G.; Grover, A.; Mastroeni, D.; Walker, D.G.; Caselli, R.J.; Kukull, W.A.; McKeel, D.; Morris, J.C. Gene expression profiles in anatomically and functionally distinct regions of the normal aged human brain. Physiological genomics 2007, 28, 311-322, https://doi.org/10.1152/physiolgenomics.00208.2006.

31. Hokama, M.; Oka, S.; Leon, J.; Ninomiya, T.; Honda, H.; Sasaki, K.; Iwaki, T.; Ohara, T.; Sasaki, T.; LaFerla, F.M. Altered expression of diabetes-related genes in Alzheimer's disease brains: the Hisayama study. Cerebral cortex 2014, 24, 2476-2488, https://doi.org/10.1093/cercor/bht101.

32. Ohara, T.; Ninomiya, T.; Kubo, M.; Hirakawa, Y.; Doi, Y.; Hata, J.; Iwaki, T.; Kanba, S.; Kiyohara, Y. Apolipoprotein genotype for prediction of Alzheimer's disease in older Japanese: the Hisayama Study. Journal of the American Geriatrics Society 2011, 59, 1074-1079, https://doi.org/10.1111/j.15325415.2011.03405.x.

33. McKay, E.C.; Beck, J.S.; Khoo, S.K.; Dykema, K.J.; Cottingham, S.L.; Winn, M.E.; Paulson, H.L.; Lieberman, A.P.; Counts, S.E. Peri-infarct upregulation of the oxytocin receptor in vascular dementia. Journal of Neuropathology \& Experimental Neurology 2019, 78, 436-452, https://doi.org/10.1093/jnen/nlz023.

34. Van der Peer Lab; http://bioinformatics.psb.ugent.be/webtools/Venn/

35. Kanehisa, M. Toward understanding the origin and evolution of cellular organisms. Protein Science 2019, 28, 1947-1951, https://doi.org/10.1002/pro.3715. 
36. Gabbouj, S.; Ryhänen, S.; Marttinen, M.; Wittrahm, R.; Takalo, M.; Kemppainen, S.; Martiskainen, H.; Tanila, H.; Haapasalo, A.; Hiltunen, M. Altered insulin signaling in Alzheimer's disease brain-special emphasis on PI3K-Akt pathway. Frontiers in neuroscience 2019, 13, 629, https://doi.org/10.3389/fnins.2019.00629.

37. Curtis, D.; Bandyopadhyay, S. Mini-review: Role of the PI3K/Akt pathway and tyrosine phosphatases in Alzheimer's disease susceptibility. Annals of Human Genetics 2021, 85, 1-6, https://doi.org/10.1111/ahg.12410.

38. Shu, Y.; Zhang, H.; Kang, T.; Zhang, J.-j.; Yang, Y.; Liu, H.; Zhang, L. PI3K/Akt signal pathway involved in the cognitive impairment caused by chronic cerebral hypoperfusion in rats. PLoS One 2013, 8, e81901, https://doi.org/10.1371/journal.pone.0081901.

39. Perluigi, M.; Pupo, G.; Tramutola, A.; Cini, C.; Coccia, R.; Barone, E.; Head, E.; Butterfield, D.A.; Di Domenico, F. Neuropathological role of PI3K/Akt/mTOR axis in Down syndrome brain. Biochimica et Biophysica Acta (BBA)-Molecular Basis of Disease 2014, 1842, 1144-1153, https://doi.org/10.1016/j.bbadis.2014.04.007.

40. Abyadeh, M.; Gupta, V.; Chitranshi, N.; Gupta, V.; Wu, Y.; Saks, D.; Wander Wall, R.; Fitzhenry, M.J.; Basavarajappa, D.; You, Y.; Salekdeh, G.H.; Haynes, P.; Graham, S.L.; Mirzaei, M. Mitochondrial Dysfunction in Alzheimer's Disease - a Proteomics Perspective. Expert Review of Proteomics 2021, https://doi.org/10.1080/14789450.2021.1918550. 